\title{
Comparing the perception with the reality of walking in a hilly environment: an accessibility method applied to a University campus in Hong Kong
}

\author{
Guibo Sun, ${ }^{1}$ Robert Haining, ${ }^{2} \mathrm{Hui}$ Lin, ${ }^{1,3}$ Nicolas M. Oreskovic, ${ }^{4-6} \mathrm{Jie} \mathrm{He}^{7}$ \\ ${ }^{1}$ Institute of Space and Earth Information Science, The Chinese University of Hong Kong, \\ Shatin, Hong Kong; ${ }^{2}$ Department of Geography, University of Cambridge, UK; ${ }^{3}$ Department \\ of Geography and Resource Management, The Chinese University of Hong Kong, Shatin, \\ Hong Kong; ${ }^{4}$ Department of Internal Medicine, Massachusetts General Hospital, Boston; \\ ${ }^{5}$ Department of Pediatrics, Massachusetts General Hospital, Boston; ${ }^{6}$ Harvard Medical \\ School, Boston, MA, USA; ${ }^{7}$ School of Architecture, Tianjin University, China
}

\begin{abstract}
The influence of hilliness on walking behavior could be a consequence of the real effect of the local topography, but individual perception of the difficulties associated with walking in a hilly environment may also be important. Previous studies have found that people's per-
\end{abstract}

\footnotetext{
Correspondence: Hui Lin, Institute of Space and Earth Information Science, The Chinese University of Hong Kong, Fok Ying Tung Remote Sensing Science Building, Shatin, Hong Kong.

Tel: +852.3943 .6010 - Fax: +852.2603 .7470 .

E-mail: huilin@cuhk.edu.hk
}

Key words: walking, hilly environment, accessibility, perception, geographic information system.

Acknowledgements: the authors wish to acknowledge Dr. Rongrong Li, from ISEIS at CUHK, for her helpful contributions with experimental design. The authors wish to acknowledge Dr. Chao Yuan, from the School of Architecture at CUHK, for his insightful suggestions on the potential implementations in land use planning based on this study. Part of the work for this paper was completed whilst Guibo Sun was a Visiting Scholar in the Department of Geography, the University of Cambridge, UK.

Funding: this study is partially supported by program Introducing Talents of Discipline to Universities (B13011). Guibo Sun and Jie He are supported by a National Natural Science Foundation of China (51478300). Nicolas Oreskovic is supported by a grant from the National Heart, Lung, and Blood Institute (K23HL103841).

Received for publication: 26 February 2015.

Revision received: 15 March 2015.

Accepted for publication: 17 March 2015.

(C) Copyright G. Sun et al., 2015

Licensee PAGEPress, Italy

Geospatial Health 2015; 10:340

doi:10.4081/gh.2015.340

This article is distributed under the terms of the Creative Commons Attribution Noncommercial License (by-nc 3.0) which permits any noncommercial use, distribution, and reproduction in any medium, provided the original author(s) and source are credited. ceptions do not necessarily match well with the realities of walking in hilly environments. There are a few methods that can be used to visualize the geography of that difference for use by urban planners and public health practitioners. A walking accessibility measure that allows comparison of perception and reality is proposed and implemented in this study. We note that difficulties in calculating accessibility measures in the present context arise primarily from problems with data quality, three-dimensional pedestrian network modelling and the adequacy of accessibility methods for describing and predicting walking behavior. We present practical strategies for addressing these issues using geographic information systems. Our method is illustrated by calculating accessibility for a hilly university campus in Hong Kong. Walking behaviors on, and people's perceptions of, this hilly environment were obtained through walking diaries and a survey. The article concludes with suggested directions for the future development of walking accessibility measures along with some ideas about their applicability to the practice of planning and designing a walkable environment.

\section{Introduction}

Walking accounts for a large portion of what is termed moderate physical activity (Bassett et al., 2000) and has many known health benefits, such as improvements to cardiovascular health, lung function, bone strength, mental health, including a decreased risk of diabetes, obesity, cancer, and overall mortality. Local topography, a neighbourhood's natural environment such as whether it is hilly or not, may play an important role in an individual's decision to be physically active. Studies seldom assess the impact of hills within the local natural environment on the attractiveness of walking. Rodriguez and Joo (2004) find that local topography is one of the factors significantly associated with the attractiveness or otherwise of non-motorized modes of transport (e.g., walking and bicycling). Slope is a major determinant of physical inactivity, subjects (especially the obese group) spend less time walking in hilly environments.

Physical activity not only depends on the real environment but also on how individuals see their walking environment, since the perceived effort of uphill walking is greater compared to level movement. While little agreement was found when objective measures were compared to respondent's perceptions in hilly neighbourhoods, it has been shown 
that real distances are often overestimated when routes cross barriers such as hills (Sadalla and Staplin, 1980). Research to date has attempted to quantify the agreement between perceived and objective measures of the natural environment, e.g., by taking into account weather conditions, the presence of trees for shade, and hilliness when assessing associations with physical activity. McGinn and her colleagues (2007) find that perceived measures exert greater impact on physical activity than their objective counterparts. They proposed that people's perception of the natural environment, including hilliness, should be taken into account when developing physical activity interventions. Environmental barriers in hilly terrain, therefore, should be considered when planning a neighbourhood that is designed to be walkable. A better understanding of the difference between perception and reality when walking in hilly environments, and being able to visualize that difference for the benefit of urban planners and public health practitioners, may lead to more effective promotion of walking behavior.

According to Hansen (1959) accessibility is a measurement of the spatial distribution of activities about a point, adjusted for the ability and the desire of people to overcome spatial separation. Indeed, it is a measure that integrates land use and transport (Geurs and Van Wee, 2004) and accessibility maps can assist urban planners in the spatial arrangement of facilities and services. However, whilst previous accessibility measures have mostly focused on motorized transport at regional scales, walking using a pedestrian network usually takes place at the finer scale of the neighbourhood. This, we argue, means walking accessibility measures need to be constructed differently from motorized ones. Few studies have focused on walking accessibility measurement, and even fewer take into account the hilliness of the neighbourhood environment. Kwan and Lee (2005) and Lee (2007) point to the necessity of working with the three-dimensional (3D) structure of the pedestrian network in a built environment for effective emergency responses. However, the speed, extent and fidelity of 3D realizations of pedestrian networks remain limited (Thill et al., 2011). Analytical methods for 3D problems, such as shortest route and accessibility analysis, have been slow to develop. We show that a practical data model can be implemented within a geographic information system (GIS) environment without difficulty.

In this paper, we develop a practical walking accessibility measure in a hilly environment combining the gravity-based approach of Hansen (1959) with that of Black and Conroy (1977), i.e. cumulative opportunities accessibility modelling. A university campus in Hong Kong was chosen as our study site to illustrate our methodology. The walking impedance values were calculated using real data from walking diaries and perception data from a questionnaire survey. A map of the differences in accessibility between the perception and reality of walking was calculated. This map might form an empirical foundation for urban planners and health practitioners to help them anticipate the effectiveness of their interventions and make policy decisions.

\section{Materials and Methods}

\section{Study area and population}

The Chinese University of Hong Kong (CUHK) was chosen as the study location. Similar to the situation found in other hilly cities, the pedestrian network on this university campus possesses a complex 3D topological layout. Express lifts, outdoor escalators, footbridges and hilly stairs on the CUHK campus directly link three height levels (from 0 to $157 \mathrm{~m}$ ). Thus, vertical movements in stairwells and elevators, oblique movements on escalators and stairs exist in this hilly commu- nity. The steeply contoured site has resulted in the dispersal of buildings and the formation of seven colleges each being a congenial neighbourhood with its own hostels, dining halls and other facilities.

University students matriculated at the university were eligible to participate. A convenience sample of students was recruited by mail and asked to complete a walking diary and a questionnaire. One-hundred and sixty-nine subjects completed the survey in December 2012. The sample had the proportion of $56 \%$ females and $44 \%$ males, which is consistent with the gender distribution at the Chinese University of Hong Kong.

\section{Walking diary: reality of walking}

A walking-oriented travel dairy, which collected information on the time of each walking trip's start and end and the route followed, was developed for this study. A campus map with a detailed pedestrian network was distributed to each subject. Daily walking trips were obtained from the walking diary and mapped in a GIS (ArcGIS 10.0, ESRI, Redlands, CA, USA) according to road segment numbers. Each subject could have multiple walking or bus transportation trips throughout their daily activities, thus providing repeated data measurements, or, one-to-many data as classified in ArcGIS. We conducted a Table Join by the one-to-many relationship class in ArcGIS. Three walking behavior outcomes were created using these data: time, distance and altitude change. We supposed the walking time to be a function of walked distance and walked altitude change (Crompton, 2006). Multivariate linear regression models controlling for gender were run to test for the hypothesized relationships with significance set at $\mathrm{P}<0.05$.

\section{Questionnaire: perception of walking time}

Subjects' perception of the neighbourhood's environment was assessed through questions on whether or not hilliness was a problem in their daily walking on campus (on a 1-to-7 disagree-agree response scale). Additionally, respondents were asked if they would use escalators and express lifts to traverse different height levels on campus (on a 1-to-7 unlikely-likely response scale).

Subjects were asked to estimate how long they thought it would take them to walk from the lower level (around $0 \mathrm{~m}$ in height) to the middle level (around $110 \mathrm{~m}$ ), and from the middle level to the upper level (around $157 \mathrm{~m}$ ). We obtained the ratio between the mean perceived and the mean real or actual walking time (RPR). There is little empirical evidence to identify the slope threshold above which people perceive hilliness to be a barrier to walking. In this study, we adopted the threshold of slope suggested by Troped et al. (2001), while using a finer grid segmentation in order to capture more details about changes in hilliness in the pedestrian network across our study area. Specifically, GIS elevation data for the CUHK campus were converted into a $50 \times 50$ m squares, which were then classified into two levels: $<5.71$ and $\geq 5.71$ degrees (equivalent to $<5$ and $\geq 5 \%$ slope, respectively). Road segments of the pedestrian network on campus where slopes exceed 5.71 degrees (perceived as a barrier when walking) were multiplied by the ratio RPR, while road segments with slopes less than 5.71 degrees (perceived not as a barrier when walking) retained their actual walking time values. These rules were used to derive estimates of the perceived walking time on campus.

\section{D pedestrian network construction in a geographic information system}

It should be noted that the pedestrian network for walking is quite different from the street network for driving on the university campus. The use of a traditional GIS street network database would be too 
coarse and inadequate to represent pedestrian paths on the campus. Ideally, pedestrian networks should incorporate formal and informal paths, including sidewalks, lanes, pedestrian bridges and park paths that are informal but frequently used by walkers. The missing pedestrian paths in the street network database are likely to be the ones that are most frequently used, and which greatly increase the connectivity of dispersed destinations. Most studies into accessibility and connectivity that only use street networks in their analyses provide an incomplete description and prediction of walking behavior (Chin et al., 2008). In this study, we develop a detailed pedestrian network dataset in addition to the use of the traditional street network. This was done by digitizing CUHK campus maps supplemented by field surveys (examples are shown in Figure 1).

A 3D pedestrian network was created by the interpolate shape (3D Analyst) tool in ArcToolbox with inputs that make use of a two-dimensional (2D) outdoor pedestrian network and a digital elevation model of the campus. The express lifts, the foot bridges between buildings and the hilly stairs were captured using the Point $Z$ data mode. For example the express lift was represented using the same $\mathrm{x}$ and $\mathrm{y}$ location for the start and end points but different $\mathrm{z}$ values. Although this 3D relationship is inherently and perceptually intricate, ArcScene tool makes it easy to visualize such relationships. Two directed networks were created having exactly opposite directions (and altitude changes) for each path segment. Walking time was assigned to each directed path segment depending on the altitude change. The minimum travel cost between an origin and a destination allowing for walking uphill and downhill was then calculated (Figure 2).

\section{Accessibility evaluation for walking: perception and reality}

In this paper, we combine a gravity-based accessibility measure (Hansen, 1959) with a cumulative opportunities accessibility measure (Black and Conroy, 1977). Gravity-based measures estimate the accessibility of point or zone $i,\left(A_{i}\right)$, to all $(n)$ other destination zones. The measure can be formulated as follows, assuming a negative exponential cost function:

$$
A_{i}=\sum_{j=1}^{n} D_{j} e^{-\beta c_{i j}}
$$

where $D_{j}$ measures all the opportunities in zone $j$ and $c$ is some measure of the cost of travel between $i$ and $j$. Previous research suggests that using either time or distance as the impedance variable is acceptable. $\beta$ is the cost sensitivity parameter. $A_{i}$ is obtained by summing over all the zones $(j=1, \ldots, n)$. The negative exponential function is most commonly used as the cost (or impedance) function, because of its links with travel behavioral theory and its suitability for describing accessibility over short distances (Handy and Niemeier, 1997). In gravitybased measures, more distant opportunities are less attractive than those that are closer. In the case of trips made by bicycle or on foot, more distant opportunities are heavily discounted because of the time it takes to reach them (Kanafani, 1983; Iacono et al., 2010). The negative exponential function satisfies that requirement.

The challenge with this measure relates to difficulties in interpreting and communicating results as it combines land-use with transport elements, whilst weighting opportunities (Geurs and Van Wee, 2004). Cumulative opportunity measures evaluate accessibility in terms of the cumulative number or proportion of opportunities that can be reached within specified travel distances or times from a reference location. Accessibility can be represented graphically by drawing the cumulative distribution of the cumulative number of opportunities reached plotted against travel time or distance (Black and Conroy, 1977), which is very convenient for comparative purposes. Notice that the cumulative opportunity measure of accessibility does not discount measures of opportunity over distance (Kwan, 1998). Combining the gravity-based with the cumulative opportunities accessibility measure in our study, addresses this issue by taking into account the spatial distribution of opportunities within fixed walking distances, making the results easier to interpret and communicate.

Public open space is an important destination for walking in neighbourhoods (Koohsari et al., 2013). The spatial configuration of and access to public open space is frequently cited as a key principle in urban design guidelines. Public open space is believed to encourage physical, mental and social outcomes by promoting population physical activity, such as recreational walking (Giles-Corti et al., 2005), decreasing obesity (Coombes et al., 2010) and improving quality of life in general. In this study, walking accessibility from each building where students live or study to public open spaces on the CUHK campus was calculated to illustrate our method. The accessibility of each building was obtained by summing across all public open spaces within a fixed acceptable cost value. The accessibility measure for each building to open public spaces on campus was estimated as:

$$
A_{i}=\sum_{j=1}^{m(k)}\left(\text { Public_Open_Space }_{j}{ }^{*-\beta^{*} \text { Walking_Time }_{i j}}\right) \text { eq. } 2 .
$$
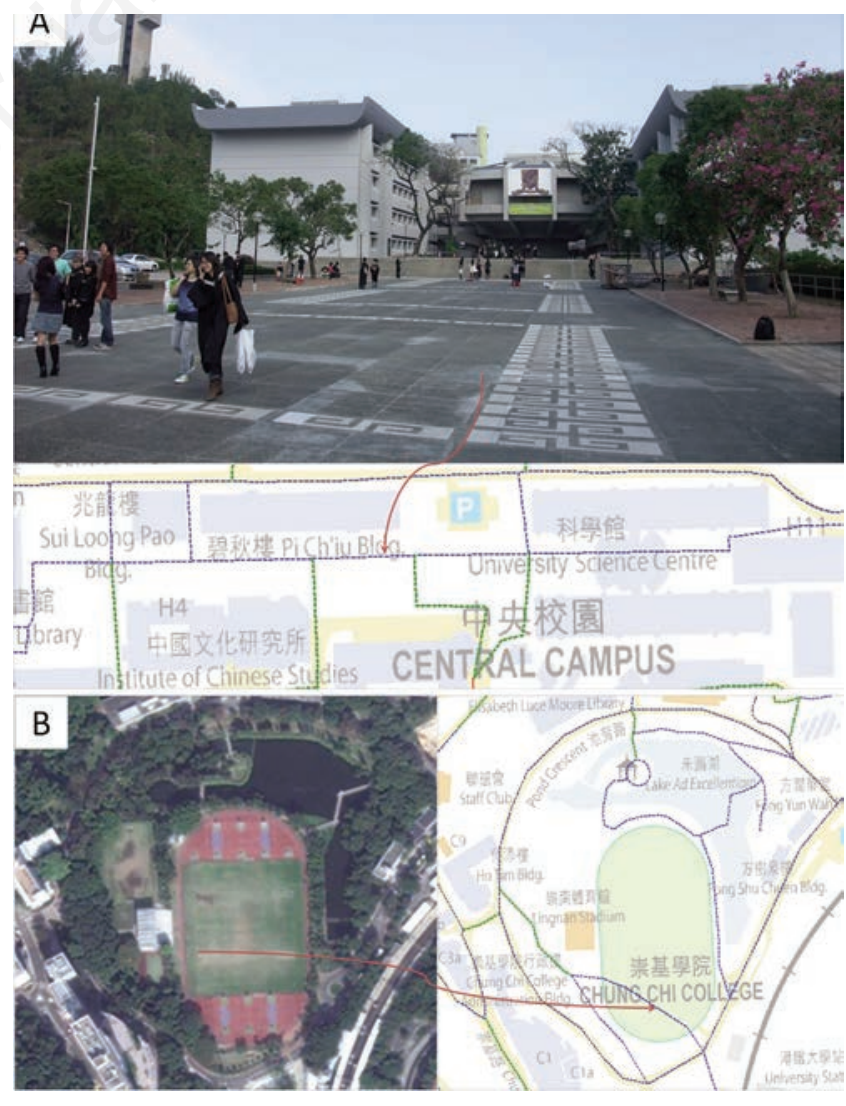

Figure 1. Examples of a detailed pedestrian network on campus. A) The central line of a public open space is used in the accessibility measure; B) a frequently used but informal road crosses the playground. 
where $m(k)$ denotes the number of accessible public open spaces within the maximum acceptable walking distance $k$. Four-hundred $\mathrm{m}$ (around a quarter of a mile) and $800 \mathrm{~m}$ (around half a mile) in real walking distances were used as the specified walking domains $(k)$ in this hilly environment (Forsyth et al., 2008). The measurement of walking accessibility was conducted using the $3 \mathrm{D}$ pedestrian network. The methodology was as follows.

Step 1, calculation of the origin-destination (0-D) cost matrix. The origin $i$ is a building and the destination $j$ a public open space. The travel cost, Walking_Time $i j$, between each 0-D pair in the matrix was based on the minimum walking time through the 3D pedestrian network distinguishing between travelling uphill, downhill, by express lift and by escalator. The $0-\mathrm{D}$ cost matrix based on the 3D pedestrian network was calculated using the Python programming language (Python $2.6,2010)$. Two types of impedance value for walking were input into the $3 \mathrm{D}$ pedestrian network. These were real walking time calculated from the walking diaries and perceived walking time obtained from the perception questionnaire and after multiplication with RPR.

Step 2, measuring the accessibility of each building. The size of the $j^{\text {th }}$ public open space, Public_Open_Space ${ }_{j}$, was used to measure how many activity opportunities are available at that destination. The parameter $\beta$ in eq. 2 for walking trips using walking time as an impedance measure was estimated from Figure $3(\beta=0.162)$. Although the function is estimated using only five data points, the data points themselves use the data from 627 measured walking trips. The accessibility for the $i^{\text {th }}$ building, $A$, was obtained by summing across all public open spaces within the two fixed walking distances of $400 \mathrm{~m}$ and $800 \mathrm{~m}$.

Step 3, interpolation to the whole community. To get an accessibility measure for any point within the study area, we interpolated these accessibility values $A_{i}$. to the whole campus using the method of inverse distance weighting. Two sets of accessibility maps using the 400 and $800 \mathrm{~m}$ walking distance thresholds were constructed.

Step 4, comparison between perception and reality for walking. We subtracted the perception accessibility map from the reality accessibility map for both the 400 and $800 \mathrm{~m}$ groups, which produced two difference maps to show the spatial distortion due to perception associated with walking in this hilly environment.

\section{Results}

\section{Reality of walking in a hilly environment}

Subject walking diaries $(\mathrm{N}=169)$ were matched to the pedestrian network map and 627 walking trips were identified. Walking time, walked distance and walked altitude changes were calculated. Descriptive statistics for the real walking data are shown in Table 1. Walking time can be predicted from these data using the multivariate linear regression model (Table 2). Walking distance $(\mathrm{P}<0.001)$ and walked altitude change $(\mathrm{P}=0.047)$ have a statistically significant association with real walking time. The real walking time of each road seg-

Table 1. Reality of walking in the hilly environment of the Chinese University of Hong Kong ( $=627$ ).

\begin{tabular}{lccc}
\hline Walking measurements & Mean & Minimum & Maximum \\
Walking time (min) & 7.69 & 2.00 & 16.00 \\
Walked distance $(\mathrm{m})$ & 536.20 & 120.08 & 1578.98 \\
\hline Walked altitude changes $(\mathrm{m})^{\circ}-6.35$ & -122.00 & 110.90 \\
\hline
\end{tabular}

${ }^{\circ}$ Positive values mean walking downhill; negative values mean walking uphill.

Table 2. Multivariate linear regression model examining the reality of walking in the hilly environment of the Chinese University of Hong Kong ( $\mathrm{N}=627)$.

\begin{tabular}{lccc} 
& \multicolumn{3}{c}{ Walking time } \\
Walked distance & 0.009 & $<0.001$ & $<0.001^{* *}$ \\
Walked altitude changes & -0.006 & 0.003 & $0.047^{*}$ \\
\hline Gender $^{\circ}$ & 0.087 & 0.286 & 0.713 \\
\hline
\end{tabular}

SE, standard error. ${ }^{\circ}$ Gender variable was coded as male $=0$ and female $=1 .{ }^{*} \mathrm{P}<0.05 ; * * \mathrm{P}<0.001$
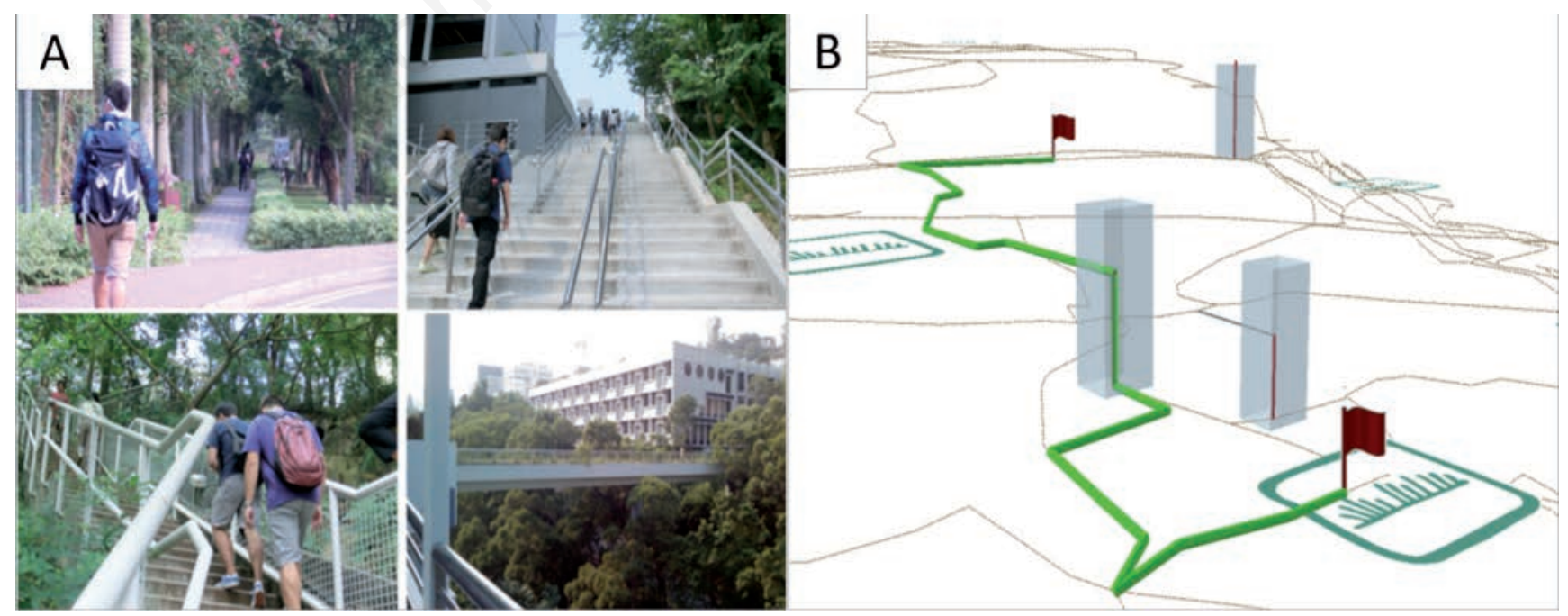

Figure 2. A three-dimensional pedestrian network. A) Pedestrian networks on campus with hilly topography; B) the 3D pedestrian network in a geographical information system (the green route represents the path with minimum travel cost). 
ment is calculated and input to the accessibility model as the reality of walking in this hilly environment.

\section{Perception of hilly environment as a barrier when walking}

The degree of agreement with statements in the questionnaire (mode) is given as a figure on a 1-to-7 scale (where the 7 denotes complete agreement), while the interquartile range (IQR) is the measure for distribution of the response to the questions. Table 3 reports descriptive statistics for the perception data ( $\mathrm{N}=159$ after deleting 10 missing data). Our results show that subjects perceive walking on hilly sidewalks as a barrier (Mode $=6, \mathrm{IQR}=1$ ), and that they would like to use escalators (Mode=7, IQR=3.8) and the express lift (Mode=7, $\mathrm{IQR}=1$ ) when traversing the hilly levels on campus.

Table 4 reports estimated, perceived walking time by subjects when they traverse the three hilly levels on campus with special reference to altitudinal direction (uphill $v s$ downhill). A landmark on each level was chosen as the specific origin or destination point at each level. From walking diaries, we calculated the real walking time between the lower level and the middle level as $15 \mathrm{~min}(\mathrm{SD}=2)$, while from the middle level to the upper level as $9 \mathrm{~min}(\mathrm{SD}=1)$. The ratio between the perceived and real walking time, was calculated and averaged (RPR $=1.46$ when walking uphill). If a road segment crosses a square with a steep slope for a continuous distance of at least $50 \mathrm{~m}$ and its slope is $\geq 5.71$ degrees, the subject is coded as having a steep hill barrier. The walking time when traversing uphill on those road segments should be multiplied by RPR, while is the same as the real walking time when walking downhill and on road segments with slopes $<5.71$ degrees. The perceived walking time for each road segment was calculated and input to the accessibility model to determine the perceived walking time in this hilly environment.

\section{Difference in accessibility by walking: perception and reality}

Figure 4 presents maps of walking accessibility (with red denoting high accessibility and blue the opposite) using the 400 and $800 \mathrm{~m}$ distance thresholds, where A1 and B1 show the real accessibility and A2 and $\mathrm{B} 2$ the perceived one. The strength of the difference between reality and perception (A3 and B3) with regard to accessibility is visualized by a shift of color towards red, which denotes greater difference. Using the $400-\mathrm{m}$ threshold, both maps show a high degree of similarity. Areas close to clusters of public open spaces are shown to have high levels of accessibility, with a gradual decline as one moves away from them. The larger cluster is located at the middle level and the smaller one at the lower level. Both of them have large amounts of public open space, and a high density of not very hilly pedestrian paths. Using the $800-\mathrm{m}$ threshold, higher overall accessibility values are seen across most of the campus. One particularly high accessibility area is found at the central campus that lies at the middle-height level. This high-accessibility location results from the clustering of public open spaces as well as proximity to the other height levels, which means that it is well placed for walking in this hilly topography. The area with the best accessibility shifts a little towards the upper level in the center-east of the maps. This may be because of the express lift installed in the center-east of the campus connecting the middle and upper levels and thus reducing the impact of hilliness on walking.

The areas with the largest difference (between perception and reality) in accessibility for walking using the $400 \mathrm{~m}$ walking threshold are identified in Figure 4 A3. The place where the difference is greatest coincides with a newly established college. This implies that the new
Table 3. Perception of hilly environment as a barrier $(\mathrm{N}=159)$.

\begin{tabular}{|c|c|c|c|}
\hline & Mean & Mode $^{\circ}$ & \\
\hline Hilly sidewalks perceived as barriers to walking & 5.15 & 6 & 1 \\
\hline Escalators perceived as helpful for traversing hilly areas & 5.02 & 7 & $3.8^{\#}$ \\
\hline Express lifts perceived as helpful for traversing hilly levels & 6.05 & 7 & 1 \\
\hline \multicolumn{4}{|c|}{ 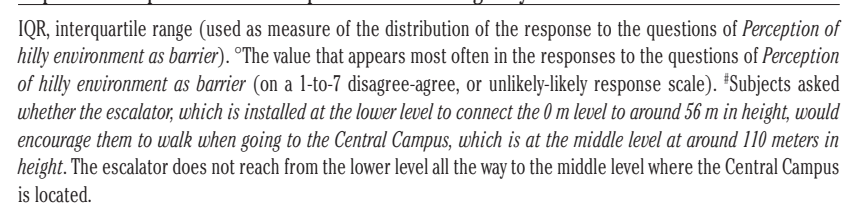 } \\
\hline
\end{tabular}

Table 4. Estimated walking time when traversing the hilly levels $(\mathrm{N}=159)$.

\begin{tabular}{lccc} 
& $\begin{array}{l}\text { Mean } \\
\text { (min) }\end{array}$ (min) & \\
$\begin{array}{l}\text { Uphill: perceived time from lower level } \\
\text { to middle level }\end{array}$ & 20.90 & 20 & 10 \\
$\begin{array}{l}\text { Downhill: perceived time from middle level } \\
\text { to lower level }\end{array}$ & 15.41 & 15 & 5 \\
\hline $\begin{array}{l}\text { Uphill: perceived time from the middle level } \\
\text { to upper level }\end{array}$ & 11.54 & 10 & 5 \\
$\begin{array}{l}\text { Downhill: perceived time from the upper level } \\
\text { to middle level }\end{array}$ & 13.78 & 10 & 5 \\
\hline IQR, interquartile range. Lower level, University station around 0 height in m; middle level, University
\end{tabular}

library around 110 height in $\mathrm{m}$; upper level, New Asia library around 157 height in $\mathrm{m}$.
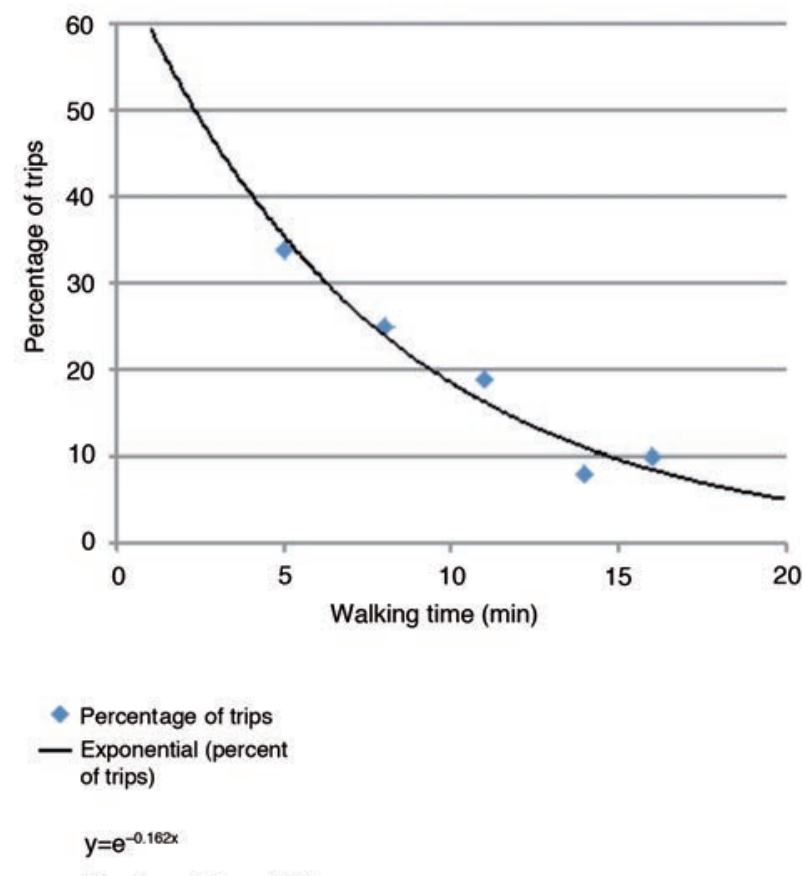

Number of trips $=627$

Figure 3. Estimation of the paramater $\beta$ for the impendance function. 
college is not well placed for accessing available facilities. Using the 800 -m walking threshold, the area of greatest difference between perception and reality is located between the lower level and the middle level (Figure 4 B3). This area is not close to any public open space and has a high density of hilly pedestrian paths.

\section{Discussion}

In this study, we proposed and developed a practical walking accessibility measure using 3D pedestrian network modelling that we applied at the CUHK to compare the perception with the reality of walking in a hilly environment. Specifically, we found that walking in a hilly environment is perceived as a barrier. Our findings are consistent with previous literature showing that the natural environment including local topography may influence people's perception and then determine their preference for direction when walking (McGinn et al., 2007). The subjects in our study preferred to use the express lift and escalators when traversing between different levels. The increase in pedestrian facilities such as installation of express lifts may increase people's willingness to walk in a hilly environment. We found a lack of correspondence between perception and reality when walking in this environment. Subjects seemed to overestimate the walking time when they walked uphill, which was consistent with their statements that walking in a hilly environment was a barrier. The overestimation of distance may be a result of distortions in awareness and knowledge of the built environment as well as an indication as to why subjects may not necessarily make frequent use of certain destinations (Golledge, 1997).

Evidence from our GIS-based fine pedestrian network construction, which contained both formal and informal routes, showed that the informal network cannot be neglected as it may have a significant
A1
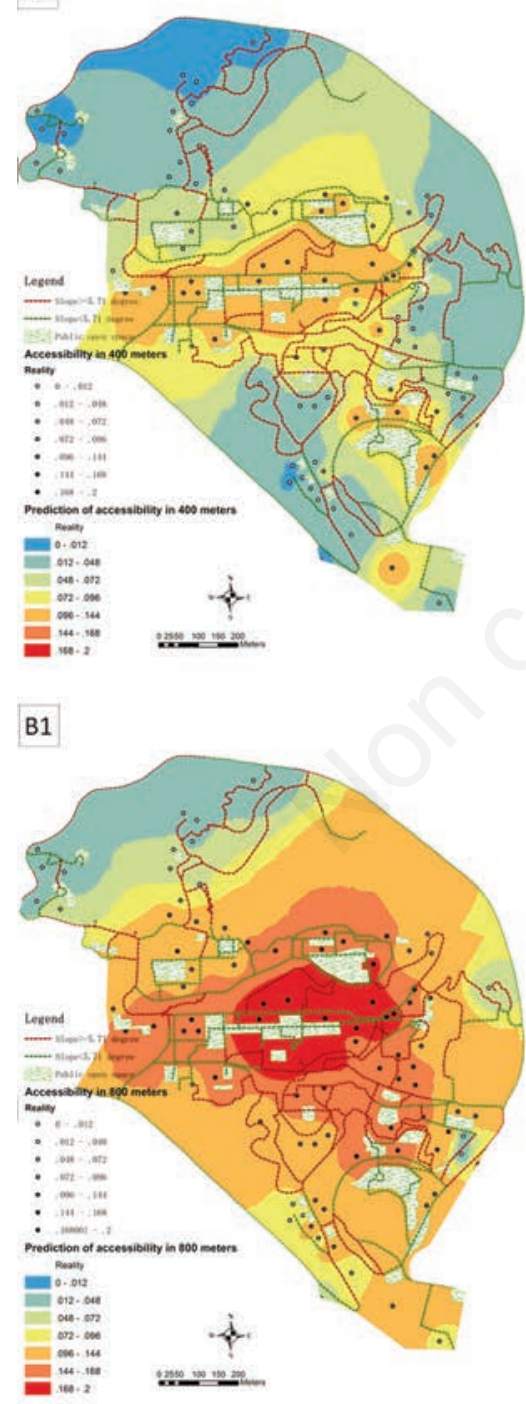

A2

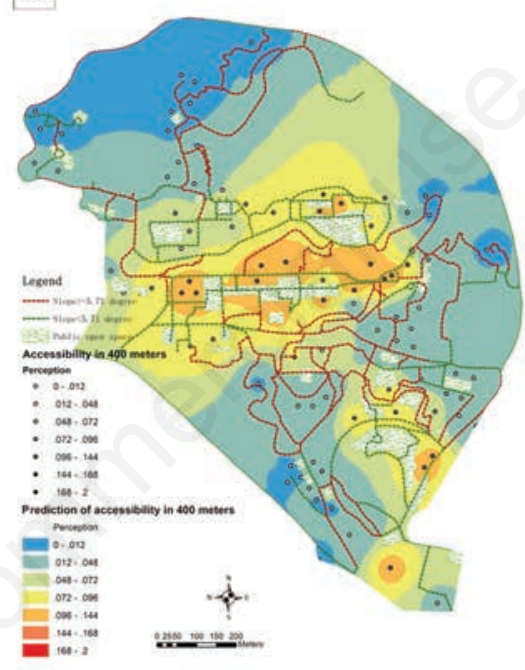

B2

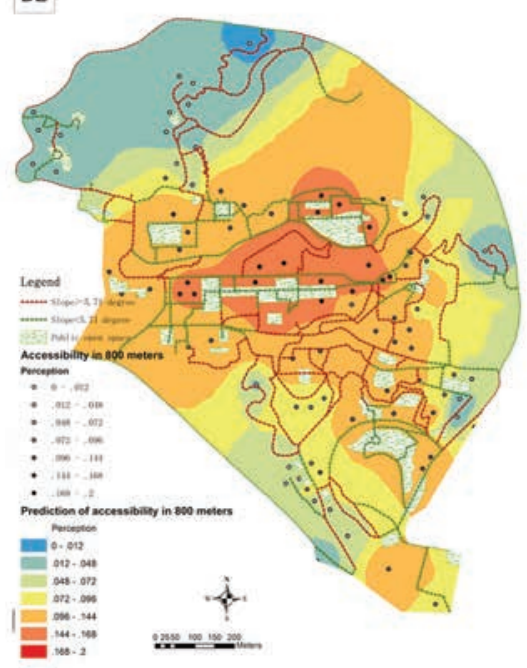

A3

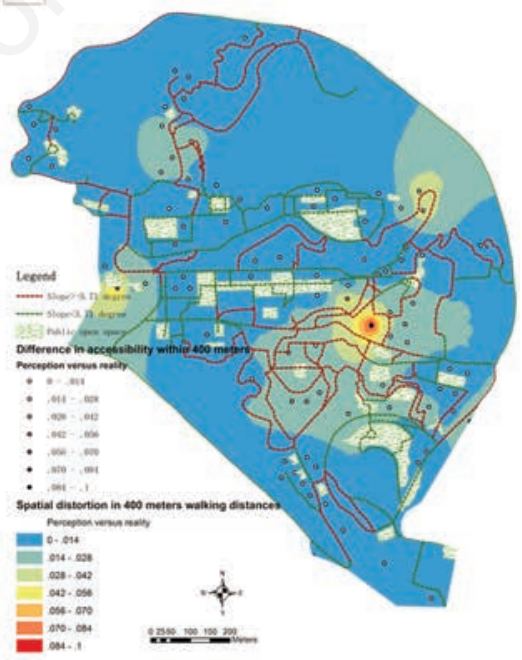

B3

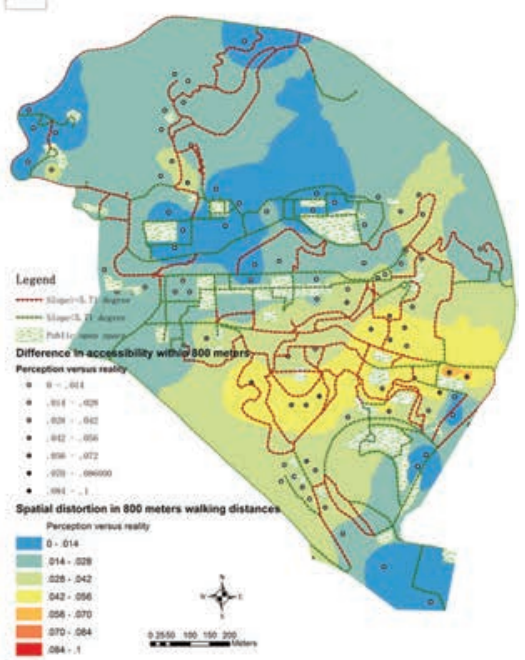

Figure 4. Walking accessibility to public open space within $400 \mathrm{~m}$ (A1, A2, A3) and $800 \mathrm{~m}(\mathrm{~B} 1, \mathrm{~B} 2$, B3). Walking accessibility in reality (A1 and B1), walking accessibility in perception (A2 and B2), and the difference between perception and reality (A3 and B3). 
influence on connectivity because of frequency of usage. Our study supports the statement of Chin et al. (2008) about the impact of missing pedestrian data in physical activity studies and the need to pay more attention to the construction of a complete pedestrian network for behavioral studies with respect to walking. Quite innovatively, the complete pedestrian network was built in a 3D layout and this we believe supports efficient computation and greater accuracy. We expect that our study will promote awareness of the importance of 3D network analysis in small-area research, such as in a building, a neighbourhood or a community, and that it may have wider utility at larger scales for transport geography and urban studies as discussed by Lee (2007). The method we have developed could be extended to other cities, with similar hilly topography for walking, and other health behavioral studies.

We have developed a practical walking accessibility measure by integrating gravity-based and cumulative opportunity measures, in order to evaluate the combined effect of land-use and walking in a hilly environment. The ability to visualize results in a GIS makes the measure more interpretable and easier to communicate. In this study, we took access to public open space as the example to illustrate the methodology. However, it can be easily extended to other accessibility analyses, such as residence-shopping and spatial configuration of healthcare facilities, especially for communities with high-rise buildings and a hilly topography.

With our walking accessibility measure, we can compare the difference between the perception and reality of walking in a hilly environment. The real and perceived assessments should be given equivalent consideration. Our maps showing the difference in accessibility between perception and reality spatially visualize the distortions in people's cognitions. Previous studies into cognitive maps are more based on polygon geographical features, while our spatial distortion polyline maps constitute an improvement in the field of spatial visualization of cognition. The maps may provide empirical evidence for urban planners and public health practitioners, when they take policy decisions aimed to promote walking. For example, there is an area between the lower level and the middle level on the CUHK campus which has relatively low accessibility to public open space (depicted in A3 and B3 in Figure 4). We suggest that new public open spaces are needed in those underserved areas, or that improvements to the pedestrian infrastructure (such as express lifts and escalators) be made. Either approach could be employed to address the stated goal of improving access.

The link between the built environment and walking behavior is of growing interest in the fields of urban planning and public health. But there still seems to be a gap between how people use, and how urban designers believe that people use, the built environment. Insufficient attention is paid to people's perceptions resulting in an underestimation of the influence of topographical features with respect to walking behavior. One consequence of this is that the presence of some facilities, such as public open spaces, in a neighbourhood or a city does not necessarily imply that residents will use them (Jacobs, 1961). It would be a reasonable endeavour to build a simple but practical platform such as we have developed in this paper, taking the difference between the perception and reality to assist urban planners and public health practitioners, whilst also taking into account the range of different types of users (for example, in terms of age or mobility).

This study could have an important implementation in land use planning if a walkable environment is being addressed. Standards and guidelines for land use planning could be modified based on whether the perception of walking accessibility matches with the reality. In scenario 1 , in a place with a high walking accessibility, and where the perception matches with the reality, we could design this place with facil- ities (e.g., commercial malls) that can be frequented by the public in order to encourage walking, and set a high density standard for that type of land use. In scenario 2, we might have a place where the perception of its walking accessibility is low, but the reality of walking accessibility is high. In this case, we could plan a personalized land use, such as residential use, for these places. Residents living in such a geographic area are likely to utilize the area frequently by walking to different parts of the place. This could improve the understanding the meaning of the real walking accessibility in that place, and allow better management of the risks of poor land use development. In scenario 3, in a place where the perception of a low walking accessibility matches with the reality, we could design this area for seldom-used functions, such as warehouses or car parking.

\section{Limitations and strengths of the study}

The study has several limitations. First, our walking diary was not validated by us, though it was adapted from established travel diaries (Parkes and Thrift, 1980) and assessed for validity by colleagues in Urban Planning and Geography at CUHK and pilot tested on volunteers. Second, our assumption of shortest-path routes may not hold for leisure walking, where keeping travel cost to a minimum may not be an important criterion. Third, a single impedance function was estimated empirically. The functional form may differ for different population subgroups and vary across locations. Finally, the study site included a single university in Hong Kong and findings may not be possible to generalise. Subjects in our study were 18 to 20 -year old students; their age will certainly have influenced their perceptions of walking in hilly environments making it necessary to also study other age groups. These problems might be exacerbated in studies, where the researcher is dealing with more heterogeneous populations. Despite these limitations, our study has important strengths. To our knowledge, few studies have compared perception and reality using maps in order to express differences geographically. Statistical testing typically reports mean difference values along with standard deviations. While such analytic methods that use aggregated data are powerful tools for identifying population-level differences between perception and reality, they lack the ability to support urban planners and public health practitioners in a practical way. Maps offer the possibility of interpreting spatial distortions through visualization. In addition, there are also few studies on walking accessibility measures (lacono et al., 2010), especially taking the hilliness of the topography into consideration. With a growing level of interest in walking behavior in urban planning and public health, detailed and robust accessibility measures geared to walking provide an additional option to form and evaluate land use and transport planning.

\section{Conclusions}

The focus on difference between perception and reality developed in this paper should assist urban planners and public health practitioners in reducing the mismatch between these two entities with regard to walking accessibility, allowing better risk management of land use development. Maps visualize spatial distortions and facilitate identification of differences between perception and reality, a better understanding of which would help urban planners and public health practitioners reduce physical and psychological barriers to walking. Programs promoting the health benefits of walking are essential for making further progress in the effort to build healthier and more liveable communities. 


\section{References}

Bassett DR, Ainsworth BE, Swartz AM, Strath SJ, Brien WLO, King GA, 2000. Validity of four motion sensors in measuring moderate intensity physical activity. Med Sci Sport Exer 32:471-80.

Black J, Conroy M, 1977. Accessibility measures and the social evaluation of urban structure. Environ Plann A 9:1013-31.

Chin GK, Van Niel KP, Giles-Corti B, Knuiman M, 2008. Accessibility and connectivity in physical activity studies: the impact of missing pedestrian data. Prev Med 46:41-5.

Coombes E, Jones AP, Hillsdon M, 2010. The relationship of physical activity and overweight to objectively measured green space accessibility and use. Soc Sci Med 70:816-22.

Crompton A, 2006. Perceived distance in the city as a function of time. Environ Behav 38:173-82.

Forsyth A, Hearst M, Oakes JM, Schmitz KH, 2008. Design and destinations: factors influencing walking and total physical activity. Urban Stud 45:1973-96.

Geurs KT, Van Wee B, 2004. Accessibility evaluation of land-use and transport strategies: review and research directions. J Transp Geogr 12:127-40.

Giles-Corti B, Broomhall MH, Knuiman M, Collins C, Douglas K, Ng K, Lange A, Donovan RJ, 2005. Increasing walking: how important is distance to, attractiveness, and size of public open space? Am J Prev Med 28:169-76.

Golledge RG, 1997. Spatial behavoir: a geographical perspective. Guilford Press, New York, NY, USA.

Handy SL, Niemeier DA, 1997. Measuring accessibility: an exploration of issues and alternatives. Environ Plann A 29:1175-94.

Hansen WG, 1959. How accessibility shapes land use. J Am Plann Assoc 25:73-6.

Iacono M, Krizek KJ, El-Geneidy A, 2010. Measuring non-motorized accessibility: issues, alternatives, and execution. J Transp Geogr 18:133-40.
Jacobs J, 1961. The death and life of great American cities. Random House LLC, New York, NY, USA.

Kanafani A, 1983. Transportation demand analysis. McGraw-Hill, New York, NY, USA.

Koohsari MJ, Kaczynski AT, Giles-Corti B, Karakiewicz JA, 2013. Effects of access to public open spaces on walking: is proximity enough? Landscape Urban Plan 117:92-9.

Kwan MP, 1998. Space-time and integral measures of individual accessibility: a comparative analysis using a point-based framework. Geogr Anal 30:191-216.

Kwan MP, Lee J, 2005. Emergency response after 9/11: the potential of real-time 3D GIS for quick emergency response in micro-spatial environments. Comput Environ Urban 29:93-113.

Lee J, 2007. A three-dimensional navigable data model to support emergency response in microspatial built-environments. Ann Assoc Am Geogr 97:512-29.

McGinn AP, Evenson KR, Herring AH, Huston SL, 2007. The relationship between leisure, walking, and transportation activity with the natural environment. Health Place 13:588-602.

Parkes D, Thrift NJ, 1980. Times, spaces, and places: a chronogeographic perspective. Wiley, Chichester, UK.

Rodriguez DA, Joo J, 2004. The relationship between non-motorized mode choice and the local physical environment. Transport Res DTR E 9:151-73.

Sadalla EK, Staplin LJ, 1980. The perception of traversed distance intersections. Environ Behav 12:167-82.

Thill JC, Dao THD, Zhou Y, 2011. Traveling in the three-dimensional city: applications in route planning, accessibility assessment, location analysis and beyond. J Transp Geogr 19:405-21.

Troped PJ, Saunders RP, Pate RR, Reininger B, Ureda JR, Thompson SJ, 2001. Associations between self-reported and objective physical environmental factors and use of a community rail-trail. Prev Med 32:191-200. 